\title{
Findings of the WMT 2018 Shared Task on Automatic Post-Editing
}

\author{
Rajen Chatterjee $^{(1)}$, Matteo Negri ${ }^{(1)}$, Raphael Rubino ${ }^{(2)}$, Marco Turchi ${ }^{(1)}$ \\ (1) Fondazione Bruno Kessler, Trento, Italy \\ (2) Universität des Saarlandes \& DFKI, Saarbrücken, Germany \\ \{chatterjee, negri,turchi\}@efbk.eu \\ $\{r a p h a e l \cdot r u b i n o\} @ d f k i \cdot d e$
}

\begin{abstract}
We present the results from the fourth round of the WMT shared task on MT Automatic Post-Editing. The task consists in automatically correcting the output of a "black-box" machine translation system by learning from human corrections. Keeping the same general evaluation setting of the three previous rounds, this year we focused on one language pair (English-German) and on domainspecific data (Information Technology), with MT outputs produced by two different paradigms: phrase-based (PBSMT) and neural (NMT). Five teams submitted respectively 11 runs for the PBSMT subtask and 10 runs for the NMT subtask. In the former subtask, characterized by original translations of lower quality, top results achieved impressive improvements, up to -6.24 TER and +9.53 BLEU points over the baseline "do-nothing" system. The NMT subtask proved to be more challenging due to the higher quality of the original translations and the availability of less training data. In this case, top results show smaller improvements up to -0.38 TER and +0.8 BLEU points.
\end{abstract}

\section{Introduction}

The WMT shared task on MT Automatic PostEditing (APE), this year at its fourth round, aims to evaluate systems for the automatic correction of errors in a machine-translated text. As pointed out by (Chatterjee et al., 2015), from the application point of view the task is motivated by its possible uses to:

- Improve MT output by exploiting information unavailable to the decoder, or by per- forming deeper text analysis that is too expensive at the decoding stage;

- Cope with systematic errors of an MT system whose decoding process is not accessible;

- Provide professional translators with improved MT output quality to reduce (human) post-editing effort;

- Adapt the output of a general-purpose MT system to the lexicon/style requested in a specific application domain.

The 2018 round of the task proposed participants with the same evaluation setting of the three previous editions (Bojar et al., 2015; Bojar et al., 2016; Bojar et al., 2017), in which the output of an unknown "black box" MT engine has to be automatically corrected by learning from human revisions of translations produced by the same engine.

This year, the task focused on one language pair $^{1}$ (English-German) and, in continuity with the 2016 and 2017 rounds, on data coming from the Information Technology domain. The main novelty was represented by the use of training/test data including, for the same source sentences, translations produced by two different MT technologies: phrase-based (in continuity with 2016 and 2017) and neural (for the first time). On one side, keeping language and domain unchanged was meant to measure the technology progress over the past. On the other side, extending the evaluation to NMTderived data was meant to explore the effectiveness of APE techniques, which now migrated to the neural paradigm, to correct data obtained with the same paradigm.

In terms of participants and submitted runs, 5 teams produced respectively 11 runs for the PBSMT subtask and 10 runs for the NMT subtask.

\footnotetext{
${ }^{1}$ As opposed to the 2017 round, in which both EnglishGerman and German-English data were considered.
} 
All submissions were produced by neural APE systems. All the teams experimented with the Transformer architecture (Vaswani et al., 2017), either directly or by adapting it to the task (see Section 2.1). The two synthetic corpora provided as additional training material (see Section 2.1) were also extensively used.

In terms of results, on PBSMT data, the last year's trend is confirmed: the migration to the neural approach to APE yielded significant quality gains to the output of phrase-based MT systems. However, while in 2017 the largest improvements with respect to the baseline were respectively -4.9 TER and +7.6 BLEU, this year the distance is even larger: -6.24 TER and +9.53. On NMT data, the gains are less evident, with the largest improvements over the baseline of -0.38 TER and +0.8 BLEU.

The large difference in terms of quality gains yield by APE can be explained in several ways. One is the different amount of in-domain training data available: in the PBSMT subtask, they comprise 28,000 instances while, in the NMT subtask, they are less than $14,000 .^{2}$ Another reason is the different MT output quality in the two datasets. Indeed, TER and BLEU scores for the PBSMT test set are respectively 24.24 and 62.99 while, in the NMT test set, they reach considerably better values of 16.84 and 74.73. Altogether, these differences contributed to make the NMT subtask more challenging, participants' scores concentrated in small TER/BLEU ranges close to the baseline and the overall results harder to interpret.

\section{Task description}

Similar to previous years, participants were provided with training and development data consisting of (source, target, human post-edit) triplets, and were asked to return automatic post-edits for a test set of unseen (source, target) pairs.

\subsection{Data}

For this year's round, the APE task focused on one language pair, English-German, and on data coming from the Information Technology (IT) domain. As emerged from the previous evaluations, the selected target domain is specific and repetitive enough to allow supervised systems to

\footnotetext{
${ }^{2}$ In addition to these small in-domain training sets, which were released by the organizers over the years, participants were also provided with large synthetic corpora described in Section 2.1.
}

learn from the training set useful correction patterns that are also re-applicable to the test set.

Training and development sets consist of (source, target, human post-edit) triplets in which:

- The source (SRC) is a tokenized English sentence with length between 3 and 30 tokens;

- The target (TGT) is a tokenized German translation of the source, which is produced by a black-box system unknown to participants. Translations were produced with two different technologies, so to obtain two different subtasks and evaluation scenarios. The first subtask, in continuity with the past, focused on handling translations produced by a domain-adapted phrase-based system (PBSMT subtask). ${ }^{3}$ The second subtask (NMT subtask) focused on handling translations produced by a domain-adapted neural system. $^{4}$

- The human post-edit (PE) is a manuallyrevised version of the target, which was produced by professional translators.

Test data consists of (source, target) pairs having similar characteristics of those in the training set. Human post-edits of the test target instances are left apart to measure system performance.

For the PBSMT subtask, the training data available include: $i$ ) all the 15,000 triplets (training, development and test) released for the 2016 round of the APE task and ii) the 13,000 training and test triplets released for the 2017 round, for a total of 28,000 instances. The test set consists of 2,000 newly-released instances.

For the NMT subtask, the training and development set respectively consist of 13,442 and 1,000 triplets, while the test set comprises 1,023 instances.

\footnotetext{
${ }^{3}$ We used a phrase-based MT system trained with generic and in-domain parallel training data, leveraging prereordering techniques (Herrmann et al., 2013), and taking advantage of POS and word class-based language models.

${ }^{4}$ The NMT system was trained with generic and indomain parallel training data using the attentional encoderdecoder architecture (Bahdanau et al., 2014) implemented in the Nematus toolkit (Sennrich et al., 2017). We used bytepair encoding (Sennrich et al., 2016) for vocabulary reduction, mini-batches of 100, word embeddings of 500 dimensions, and gated recurrent unit layers of 1,024 units. Optimization was done using Adam and by re-shuffling the training set at each epoch.
} 


\begin{tabular}{l|l|l|l|l}
\hline & \multicolumn{4}{|c}{ Instances } \\
\cline { 2 - 5 } & Corpus & 2016 & 2017 & 2018 \\
\hline \multirow{3}{*}{ PBSMT } & Train & 12,000 & 11,000 & - \\
& Dev & 1,000 & - & - \\
& Test & 2,000 & 2,000 & 2,000 \\
\hline \multirow{3}{*}{ NMT } & Train & - & - & 13,442 \\
& Dev & - & - & 1,000 \\
& Test & - & - & 1,023 \\
\hline \hline \multirow{2}{*}{ Additional } & Artificial & & $4.5 \mathrm{M}$ & \\
Resources & eSCAPE-PBSMT & - & - & $7,258,533$ \\
& eSCAPE-NMT & - & - & $7,258,533$ \\
\hline
\end{tabular}

Table 1: Data statistics.

\begin{tabular}{c|c|c|c|c|c|c} 
& APE15 & APE16 & APE17 & APE17 & APE18 & APE18 \\
\hline Language & En-Es & En-De & En-De & De-En & En-De & En-De \\
Domain & News & IT & IT & Medical & IT & IT \\
MT type & PBSMT & PBSMT & PBSMT & PBSMT & PBSMT & NMT \\
\hline \hline Repetition Rate SRC & 2.905 & 6.616 & 7.216 & 5.225 & 7.139 & 7.111 \\
Repetition Rate TGT & 3.312 & 8.845 & 9.531 & 6.841 & 9.471 & 9.441 \\
Repetition Rate PE & 3.085 & 8.245 & 8.946 & 6.293 & 8.934 & 8.941 \\
\hline \hline TER $(\downarrow)$ & 23.84 & 24.76 & 24.48 & 15.55 & 24.24 & 16.84 \\
\hline BLEU $(\uparrow)$ & n/a & 62.11 & 62.49 & 79.54 & 62.99 & 74.73
\end{tabular}

Table 2: Repetition Rate and translation quality (TER/BLEU of TGT) of the WMT15, WMT16, WMT17 and WMT18 APE task data. Grey columns refer to data covering different language pairs and domains with respect to this year's evaluation round.

Participants were also provided with additional training material for both the subtasks. One resource (called "Artificial" in Table 1) is the corpus of 4.5 million artificially-generated postediting triplets used by the 2016 winning system (Junczys-Dowmunt and Grundkiewicz, 2016). This corpus was widely used by participants in the 2017 round of the APE task. The other resource is the English-German section of the eSCAPE corpus (Negri et al., 2018). It comprises 14.5 million instances, which were artificially generated both via phrase-based and neural translation (7.25 millions each) of the same source sentences.

Table 1 provides basic statistics about the data, which was released by the European Project QT21 (Specia et al., 2017).

In addition, Table 2 provides a view of the data from a task difficulty standpoint. For each dataset released in the four rounds of the APE task, we report the repetition rate of SRC, TGT and PE elements, as well as the TER (Snover et al., 2006) and the BLEU score (Papineni et al., 2002) of the TGT elements (i.e. the original target translations).

The repetition rate measures the repetitiveness inside a text by looking at the rate of non-singleton n-gram types $(n=1 \ldots 4)$ and combining them us- ing the geometric mean. Larger values indicate a higher text repetitiveness and, as discussed in (Bojar et al., 2016; Bojar et al., 2017), suggest a higher chance of learning from the training set correction patterns that are applicable also to the test set. In the previous rounds of the task, we considered the large differences in repetitiveness across the datasets as a possible explanation for the variable gains over the baseline obtained by participants. In this perspective, the low system performance observed in the APE15 task and in the APE17 German-English subtask was in part ascribed to the low repetition rate in the data. In contrast, much higher repetition rates in the data likely contributed to facilitate the problem in the APE16 task and in the APE17 English-German subtask, in which most of the participants achieved significant gains over the baseline. For this year's data, values are in line with these two previous rounds.

The TER $(\downarrow)$ and BLEU $(\uparrow)$ scores reported in Table 2 are computed using the human post-edits as reference. As discussed in (Bojar et al., 2017), numeric evidence of a higher quality of the original translations can indicate a smaller room for improvement for APE systems (having, at the same time, less to learn during training and less to cor- 


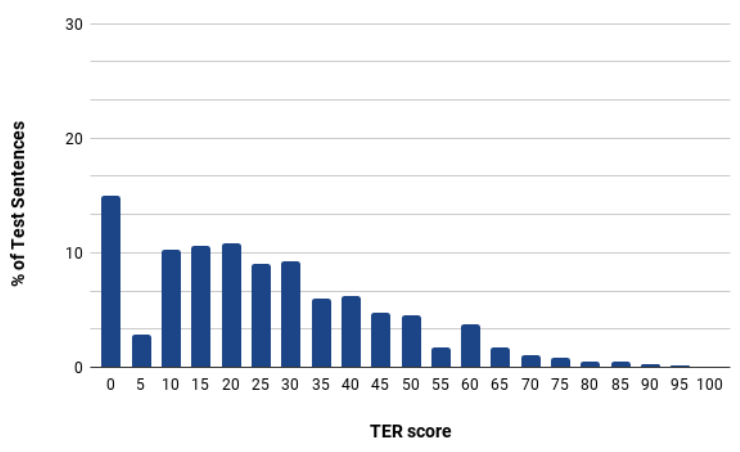

Figure 1: TER distribution in the PBSMT test set

rect at test stage). On one side, indeed, training on good (or near-perfect) automatic translations can drastically reduce the number of learned correction patterns. On the other side, testing on similarly good translations can drastically reduce the number of corrections required and the applicability of the learned patterns, thus making the task more difficult. As observed in the previous APE evaluation rounds, there is a noticeable correlation between translation quality and systems' performance. In 2016 and 2017, on EnglishGerman data featuring a similar level of quality (24.76/24.48 TER, 62.11/62.49 BLEU), the top neural systems achieved significant improvements over the baseline (-3.24 TER and +5.54 BLEU in 2016, -4.88 TER and +7.58 BLEU in 2017). In 2017, on higher quality German-English data (15.55 TER, 79.54 BLEU), the observed gains were much smaller (-0.26 TER, +0.28 BLEU). These numbers are not directly comparable since the higher quality 2017 data cover a different language pair and belong to a different domain. Nevertheless, as discussed in Section 4, this year's results confirm the correlation between the quality of the initial translations and the actual potential of APE.

Further indications about the difficulty of the two subtasks are provided by Figures 1 and 2, which plot the TER distribution for the items in the two test sets. As can be seen, the PBSMT test data are more distributed in terms of quality, with $50 \%$ of the items in the first five TER bins. Similar to last year, what makes a big difference between the two test sets is the proportion of "perfect" test instances having TER $=0$ (i.e. items that should not be modified by the APE systems). For the PBSMT subtask they are $15.0 \%$ of the total, a value similar to the APE17 English-German task in which participants achieved large baseline im-

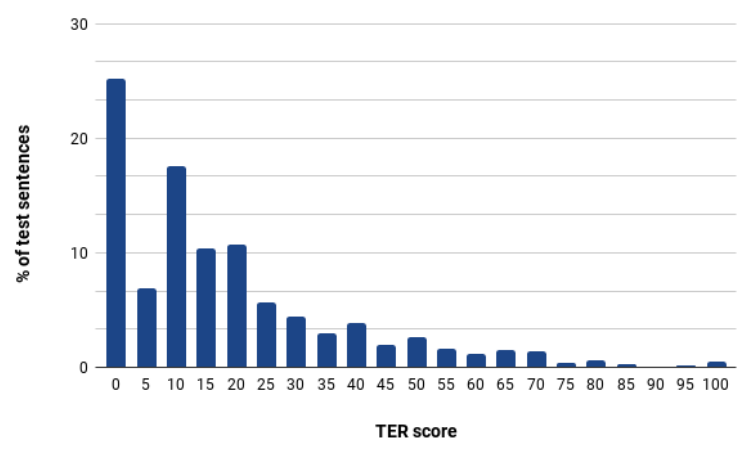

Figure 2: TER distribution in the NMT test set

provements. For the NMT subtask, they are 25.2\% of the total: much less than the proportion of the challenging APE17 German-English data (45.0\%) but still a considerably higher value compared to the PBSMT subtask. For these test items, any correction made by the APE systems will be treated as unnecessary and penalized by automatic evaluation metrics. This problem calls for conservative and precise systems able to properly fix errors only in the remaining test items, leaving the "perfect" ones unmodified.

\subsection{Evaluation metrics}

System performance was evaluated both by means of automatic metrics and manually. Automatic metrics were used to compute the distance between automatic and human post-edits of the machine-translated sentences present in the test sets (i.e. for each of the target sentences in the PBSMT and NMT test sets). To this aim, TER and BLEU (case-sensitive) were respectively used as primary and secondary evaluation metrics. Systems were ranked based on the average TER calculated on the test set by using the TERcom ${ }^{5}$ software: lower average TER scores correspond to higher ranks. BLEU was computed using the multi-bleu.perl package ${ }^{6}$ available in MOSES.

Manual evaluation was conducted via direct human assessment (Graham et al., 2016) performed by professional translators and proficient translation students, as discussed in Section 6.

\subsection{Baseline}

In continuity with the previous rounds, the official baseline results were the TER and BLEU scores

\footnotetext{
${ }^{5}$ http://www.cs. umd.edu/ s nover/tercom/

${ }^{6}$ https://github.com/moses-smt/ mosesdecoder/blob/master/scripts/ generic/multi-bleu.perl
} 


\begin{tabular}{l|l} 
ID & Participating team \\
\hline DFKI-MLT & German Research Center for Artificial Intelligence, Germany (Pylypenko and Rubino, 2018) \\
FBK & Fondazione Bruno Kessler, Italy (Tebbifakhr et al., 2018) \\
MS_UEdin & Microsoft, USA \& University of Edinburgh, Poland (Junczys-Dowmunt and Grundkiewicz, 2018) \\
POSTECH & Pohang University of Science and Technology, South Korea (Shin and Lee, 2018) \\
USAAR_DFKI & Saarland University \& German Research Center for Artificial Intelligence, Germany (Pal et al., 2018) \\
\hline
\end{tabular}

Table 3: Participants in the WMT18 Automatic Post-Editing task.

calculated by comparing the raw MT output with the human post-edits. In practice, the baseline APE system is a "do-nothing" system that leaves all the test targets unmodified. Baseline results, the same shown in Table 2, are also reported in Tables 4 and 5 for comparison with participants' submissions. ${ }^{7}$

For each submitted run, the statistical significance of performance differences with respect to the baseline was calculated with the bootstrap test (Koehn, 2004).

\section{Participants}

Five participating teams submitted a total of 11 runs for the PBSMT subtask and 10 runs for the NMT subtask. Participants are listed in Table 3, and a short description of their systems is provided in the following.

German Research Center for Artificial Intelligence - MLT group. The DFKI-MLT's participation is based on a single APE model that is jointly trained to handle PBNMT and NMT outputs. This was achieved by adding, at the beginning of every MT segment to be corrected, a specific token indicating which type of MT system was used to produce it and from which training corpus the segment pair was extracted. (i.e. the WMT training data, the artificial training data presented in (Junczys-Dowmunt and Grundkiewicz, 2016), or the eSCAPE corpus (Negri et al., 2018)). The submitted runs were obtained with two neural architectures. One ("LSTM") is an attentional RNN with gated units based on (Bahdanau et al., 2014) and implemented in OpenNMT (Klein et al., 2017). The other is the multi-head attention-only network (Vaswani et al., 2017) implemented in

\footnotetext{
${ }^{7}$ In addition to the do-nothing baseline, in previous rounds we also compared systems' performance with a reimplementation of the phrase-based approach firstly proposed by Simard et al. (2007), which represented the common backbone of APE systems before the spread of neural solutions. As shown in (Bojar et al., 2016; Bojar et al., 2017), the steady progress of neural APE technology has made the phrase-based solution not competitive with current methods reducing the importance of having it as an additional term of comparison.
}

the Marian NMT toolkit (Junczys-Dowmunt et al., 2018). For the attention-only approach, two models (i.e. "Transf.base" and "Transf.large") were trained with different configurations in terms of parallel attention layers (4 and 8 respectively).

Fondazione Bruno Kessler. FBK's system improves the multi-source neural approach adopted in (Chatterjee et al., 2017). The improvements target lower complexity of the architecture and, in turn, higher efficiency without loss in performance. To this aim, the proposed solution relies on the Transformer architecture (Vaswani et al., 2017), which was modified to incorporate multiple encoders, thereby leveraging information also from the source sentences. In addition, similar to (Hokamp, 2017), the system exploits minimumrisk training for fine-tuning (Shen et al., 2016) to avoid exposure bias and to be consistent with the automatic evaluation metrics used for the task. Finally, in order to reduce the vocabulary size, the system applies ad hoc pre-processing for the German language by re-implementing the pipeline developed by the best system at the WMT'17 Translation task (Huck et al., 2017). In addition to the data released for the task, training is performed by taking advantage of both the artificial data provided by (Junczys-Dowmunt and Grundkiewicz, 2016) and the eSCAPE corpus (Negri et al., 2018). The submitted runs, which rely on the same multi-source architecture and pre-processing step, differ in the loss function used, which is either minimum-risk training alone (" $M R T$ "), or its linear combination with maximum likelihood estimation ("MRT+MLE").

\section{Microsoft \& University of Edinburgh.} MS_UEdin's neural APE system is based on the dual-source Transformer models available in Marian (Junczys-Dowmunt et al., 2018). The models are trained with tied embeddings across all embeddings matrices and shared parameters for all the encoders. The dual-source Transformer model is implemented by stacking an additional target-source multi-head component on the previ- 
ous multi-head component, one for each encoder. Each multi-head attention block is followed by a skip connection from the previous input and layer normalization. Each encoder corresponds exactly to the implementation from (Vaswani et al., 2017), but with common parameters. The decoder consists of a self-attention block, a targetto-source attention block, another target-to-source attention block and a feed-forward network. Apart from this modification, the system follows the transformer-base configuration from (Vaswani et al., 2017). The synthetic data provided by Junczys-Dowmunt and Grundkiewicz (2016) and the eSCAPE corpus (Negri et al., 2018) were both used during training, the latter being splitted into subsets by means of domain selection algorithms aimed to isolate useful portions for the APE target domain (IT). Final submissions were produced with an ensemble of models trained on the different subsets.

Pohang University of Science and Technology. POSTECH's system is a multi-encoder model that extends the Transformer implementation in the Tensor2tensor library in order to model the relation between the original translation produced by the MT system and the ideal translation produced by the human. System training was performed by taking advantage of the synthetic data released by Junczys-Dowmunt and Grundkiewicz (2016), which were divided into a smaller (526,368 instances) and a larger sub-portion $(4,391,180)$ and used in a training process based on step-wise data reductions. The final submissions were obtained from the best single models (top-1), as well as their combination with different ensembling techniques ("fix 5" - the top-5 models in a fixed checkpoint frequency and "var5" - five top-1 models for various checkpoint frequencies).

\section{Saarland University \& German Research Cen-} ter for Artificial Intelligence. USAAR_DFKI's APE system extends the transformer-based NMT architecture by using two encoders, a joint encoder, and a single decoder. The presented model concatenates two separate self-attention-based encoders $\left(e n c_{s r c}\right.$ and $\left.e n c_{m t}\right)$ and passes this sequence through another self-attended joint encoder $\left(e n c_{s r c, m t}\right)$ to ensure capturing dependencies between $s r c$ and $m t$. Finally, this joint encoder is fed to the decoder which follows a similar architecture as described in (Vaswani et al., 2017).
A comparison between this multi-source architecture (i.e, $\{s r c, m t\} \rightarrow p e$ ), a monolingual transformer model (i.e., $m t \rightarrow p e$ ) and an ensemble of the multi-source $\{s r c, m t\} \rightarrow$ pe and singlesource $m t \rightarrow$ pe models showed better results from the ensemble model (both in the PBSMT and the NMT subtasks), which was hence used for the final submission.

\section{Results}

Participants' results are shown in Tables 4 (PBSMT subtask) and 5 (NMT subtask). The submitted runs are ranked based on the average TER (case-sensitive) computed using human post-edits of the MT segments as reference, which is the APE task primary evaluation metric ("TER (pe)"). The two tables also report the BLEU score computed using human post-edits ("BLEU (pe)" column), which represents our secondary evaluation metric. These results are commented in Section 4.1 .

The last four columns of both tables report the TER/BLEU scores computed using external references ("TER (ref)" and "BLEU (ref)") and the multi-reference TER/BLEU scores computed using human post-edits and external references ("TER (pe+ref)" and "BLEU (pe+ref)"). These results are commented in Section 4.2.

As a general remark about the two subtasks, we observe that in the NMT subtask, with all the metrics considered, the performance differences between the submitted runs are smaller (and more often not significant) compared to the PBSMT subtask. As discussed in the next sections, this makes it difficult to draw firm conclusions from the analysis of Table 5.

\subsection{Automatic metrics computed using human post-edits}

In terms of systems' ranking, the primary ("TER $(p e) ")$ and secondary evaluation metric ("BLEU (pe)") produce similar results. ${ }^{8}$ On both the subtasks, the small differences in the TER-based and BLEU-based ranking concern a different ordering of the runs submitted by specific teams: one for the PBSMT subtask (in which FBK's primary submission is slightly better than the contrastive one in terms of BLEU) and two for the NMT subtask (in which POSTECH's and DFKI-MLT's best

\footnotetext{
${ }^{8}$ The correlation between the ranks obtained by the two metrics is 0.99 for the PBSMT subtask and 0.97 for the NMT subtask.
} 


\begin{tabular}{l|l|l||l|l|l|l} 
ID & $\begin{array}{l}\text { TER } \\
\text { (pe) }\end{array}$ & $\begin{array}{l}\text { BLEU } \\
\text { (pe) }\end{array}$ & $\begin{array}{l}\text { TER } \\
\text { (ref) }\end{array}$ & $\begin{array}{l}\text { BLEU } \\
\text { (ref) }\end{array}$ & $\begin{array}{l}\text { TER } \\
\text { (pe+ref) }\end{array}$ & $\begin{array}{l}\text { BLEU } \\
\text { (pe+ref) }\end{array}$ \\
\hline MS_UEdin Primary & 18.0 & 72.52 & 42.66 & 42.93 & 17.03 & 76.7 \\
FBK Contrastive (MRT+MLE) & 18.62 & 71.04 & 43.29 & 41.99 & 17.79 & 75.19 \\
FBK Primary (MRT) & 18.94 & 71.22 & 43.74 & 41.67 & 18.18 & 74.96 \\
POSTECH Contrastive (fix5) & 19.63 & 69.87 & 43.91 & 41.46 & 18.82 & 74.02 \\
POSTECH Primary & 19.72 & 69.8 & 43.95 & 41.45 & 18.9 & 73.94 \\
POSTECH Contrastive (var5) & 19.74 & 69.7 & 43.98 & 41.35 & 18.9 & 73.93 \\
USAAR_DFKI Primary & 22.69 & 66.16 & 46.08 & 39.26 & 21.98 & 69.73 \\
USAAR_DFKI* & 22.88 & 66.05 & 46.09 & 39.27 & 22.13 & 69.68 \\
DFKI-MLT Primary (Transf.large) & $24.19 \dagger$ & 63.4 & 47.98 & 36.81 & $23.68 \dagger$ & 66.66 \\
Baseline & 24.24 & 62.99 & 48.33 & 36.42 & 23.76 & 66.21 \\
DFKI-MLT Contrastive (Transf.base) & $24.5 \dagger$ & $62.78 \dagger$ & $48.27 \dagger$ & $36.61 \dagger$ & $24.04 \dagger$ & $66.11 \dagger$ \\
DFKI-MLT Contrastive (LSTM) & 25.3 & 62.1 & $48.55 \dagger$ & $36.19 \dagger$ & 24.74 & 65.33
\end{tabular}

Table 4: Results for the WMT18 APE PBSMT subtask - average TER $(\downarrow)$, BLEU score $(\uparrow)$. The symbol " $\uparrow$ " indicates a difference from the MT baseline that is not statistically significant. The symbol "*” indicates a late submission by the USAAR_DFKI team.

\begin{tabular}{l|l|l||l|l|l|l} 
ID & $\begin{array}{l}\text { TER } \\
\text { (pe) }\end{array}$ & $\begin{array}{l}\text { BLEU } \\
\text { (pe) }\end{array}$ & $\begin{array}{l}\text { TER } \\
\text { (ref) }\end{array}$ & $\begin{array}{l}\text { BLEU } \\
\text { (ref) }\end{array}$ & $\begin{array}{l}\text { TER } \\
\text { (pe+ref) }\end{array}$ & $\begin{array}{l}\text { BLEU } \\
\text { (pe+ref) }\end{array}$ \\
\hline FBK Primary (MRT) & 16.46 & 75.53 & $42.26 \dagger$ & $44.3 \dagger$ & 16.03 & 77.36 \\
MS_UEdin Primary & 16.5 & 75.44 & $42.15 \dagger$ & $44.46 \dagger$ & 16.05 & 77.49 \\
FBK Contrastive (MRT+MLE) & 16.55 & 75.38 & $42.15 \dagger$ & $44.37 \dagger$ & 16.09 & 77.28 \\
POSTECH Contrastive (top1) & $16.7 \dagger$ & 75.14 & $42.16 \dagger$ & $44.29 \dagger$ & 16.23 & 77.16 \\
POSTECH Primary (fix5) & $16.71 \dagger$ & 75.13 & $42.2 \dagger$ & $44.21 \dagger$ & 16.23 & 77.12 \\
POSTECH Contrastive (var5) & $16.71 \dagger$ & 75.2 & $42.19 \dagger$ & $44.27 \dagger$ & 16.23 & 77.15 \\
Baseline & 16.84 & 74.73 & 42.24 & 44.22 & 16.27 & 76.83 \\
USAAR_DFKI Primary & 17.23 & 74.22 & $42.51 \dagger$ & 43.93 & 16.81 & 76.14 \\
DFKI-MLT Contrastive (Transf.base) & 18.84 & 70.87 & 43.74 & 41.53 & 18.37 & 72.93 \\
DFKI-MLT Primary (Transf.large) & 18.86 & 70.98 & 43.79 & 41.53 & 18.41 & 72.95 \\
DFKI-MLT Contrastive (LSTM) & 19.88 & 69.35 & 44.28 & 40.91 & 19.43 & 71.36
\end{tabular}

Table 5: Results for the WMT18 APE NMT subtask - average TER $(\downarrow)$, BLEU score $(\uparrow)$. The symbol " $\uparrow$ ” indicates a difference from the MT baseline that is not statistically significant.

runs in terms of BLEU are different from those produced by the TER-based ranking). In both subtasks, however, the performance differences between the submitted runs are in general quite small: in a TER interval of less than one point we have the three top submissions to the PBSMT subtask and up to six submissions to the NMT subtask. In this situation, slightly different rankings produced by the two metrics are not surprising.

PBSMT subtask. This subtask has similar characteristics to the previous APE rounds. As shown by the results of the do-nothing baseline (24.24 TER, 62.99 BLEU), the original translations in the test set have a similar quality to those of the APE16 and APE17 En-De test sets (see Table 2). In spite of this, we observe further improvements compared to last year, in which the winning system was able to beat the baseline by -4.9 TER and +7.6 BLEU points. Also this year, all participants managed to beat the MT baseline at least with their primary submission but the top-ranked submission (MS_UEdin Primary) achieved larger improvements up to -6.24 TER and +9.53 BLEU points. Moreover, three submissions out of eleven outperformed the baseline by at least -5.0 TER and +8.0 BLEU points, which suggests a positive trend in terms of technology advancements. This can also be due to the availability of new additional training data (the eSCAPE corpus). However, verifying this hypothesis would require additional ablation tests since only one team (POSTECH) did not use all the available resources.

NMT subtask. In this subtask, the situation is rather different and the higher difficulty of correcting translations of better quality (16.84 TER, 74.73 BLEU) by learning from a smaller train- 
ing set (less than half of the PBSMT subtask data) is confirmed. Results, even in the best case (FBK Primary), improve the baseline with a much smaller margin compared to the PBSMT subtask (-0.38 TER and +0.8 BLEU). Although they are obtained with the same neural technology successfully deployed for the PBSMT subtask, the majority of the scores fall in a range of less than one TER/BLEU point improvement over the baseline. Although not directly comparable, these results are in line with those of the APE17 evaluation, which was carried out on German-English phrase-based translations featuring a similar level of quality (15.55 TER, 79.54 BLEU, see Table 2). The fact that current neural APE technology performs similarly on phrase-based and neural outputs of comparable quality suggests that the quality of the machine-translated text to be corrected plays a more important role than the MT paradigm itself.

\subsection{Automatic metrics computed using external references}

By learning from (SRC, TGT, PE) triplets, APE systems' goal is to perform a "monolingual translation" from raw MT output into its correct version. In this translation process, the same sentence can be corrected in many possible ways that make the space of possible valid outputs potentially very large. Ideally, from this space, APE systems should select solutions that reflect as much as possible the post-editing style of the training data (in real-use settings, this can be the style/lexicon of specific users, companies, etc.). However, nothing prevents to end up with outputs that partially satisfy this constraint. In light of these considerations, TER and BLEU scores computed using human post-edits as reference represent a reliable measure of quality but:

1. They provide us with partial information on how systems' output reflects the post-editing style of the training data;

2. They are not informative at all about the amount of valid corrections that are not present in the human post-edits.

\subsubsection{Output style}

To gain further insights on point 1 ., the "TER (ref)" and "BLEU (ref)" columns in Tables 4 and 5 show the TER and BLEU scores computed against independent reference translations. The rational behind their computation is that differences in "TER/BLEU (pe)" and "TER/BLEU (ref)" can be used as indicators of the "direction" taken by the trained models (i.e. either towards humans' postediting style or towards a generic improvement of the MT output). Since independent references are usually very different from conservative human post-edits of the same TGT sentences, all the TER/BLEU scores measured using independent references are expected to be worse. However, if our hypothesis holds true, visible differences in the baseline improvements measured with "TER/BLEU (pe)" and "TER/BLEU (ref)" should indicate system's ability to model the post-editing style of the training data. In particular, larger gains measured with "TER/BLEU (pe)" will be associated to this desired ability.

PBSMT subtask. As can be seen in Table 4, the PBSMT subtask results show this tendency. Looking at the improvements over the baseline, those measured by computing TER and BLEU scores against human post-edits are often larger than those computed against independent references. In terms of TER, this holds true for the top six submitted runs, with the best system that shows a difference of 0.57 TER points in the gains over the baseline computed with "TER (pe)" (6.24) and those computed with "TER (ref)" (5.67). In terms of BLEU, the differences are more visible. For the top nine submissions, the baseline improvements are larger when computed with "BLEU (pe)". The best system improves over the baseline by 9.53 points with "BLEU (pe)" and 6.51 points with " $B L E U($ ref $)$ ", with a difference of 3.02 points that can be explained by its tendency to reflect the post-editing style of the training data.

NMT subtask. Similar considerations could be drawn for the NMT subtask but the small differences in the results reported in Table 5 (many of which are not statistically significant) do not allow to draw firm conclusions. For the top six submissions, TER and BLEU differences with respect to the baseline are larger when the two metrics are computed against post-edits. For the best submission, the improvements over the baseline are respectively 0.38 and 0.02 with "TER (pe)" and "TER (ref)". In terms of BLEU, they are 0.8 with "BLEU (pe)" and 0.08 with "BLEU (ref)". 


\subsubsection{Over-corrections}

To shed light on point 2., the "TER (pe+ref)" and "BLEU (pe+ref)" columns in Tables 4 and 5 show the multi-reference TER and BLEU scores computed against post-edits and independent references. The rational behind their computation is that differences in "TER/BLEU (pe)" and "TER/BLEU (pe+ref)" can be used to analyze the quality of the unnecessary corrections performed by the systems (or, in other words, to study the impact of systems' tendency towards "overcorrection"). APE corrections of a given MT output can indeed be of different types, namely: $i$ ) correct edits of a wrong passage, ii) wrong edits of a wrong passage, iii) correct edits of a correct passage and $i v$ ) wrong edits of a correct passage. TER/BLEU scores computed against human postedits work reasonably well in capturing cases $i$ )ii) by matching APE systems' output with human post-edits: for wrong MT output passages (i.e. those changed by the post-editor), they inform us about the general quality of automatic corrections (i.e. how close they are to the post-editor's actions). Cases iii)-iv), in contrast, are more problematic since any change performed by the system to a correct passage (i.e. those that were not changed by the post-editor) will always be penalized by automatic comparisons with human postedits. Although discriminating between the two types of unnecessary corrections is hard, we hypothesize that a comparison between "TER/BLEU (pe)" and "TER/BLEU (pe+ref)" can be used as a proxy to quantify those belonging to type iii). In general, due to the possibility to match more and longer $\mathrm{n}$-grams in a multi-reference setting, "TER/BLEU (pe+ref)" scores are expected to be higher than "TER/BLEU (pe)" scores. However, if our hypothesis holds true, visible differences in the increase observed for the baseline and for the systems should indicate system's tendency to produce acceptable over-corrections (type iii)). In particular, larger gains observed for the APE systems will be associated to their over-correction tendency towards potentially acceptable edits that should not be penalized by automatic evaluation metrics.

PBSMT subtask. As can be seen in Table 4, the multi-reference results computed with "TER/BLEU (pe+ref)" are unsurprisingly better than those computed with "TER/BLEU (pe)". The variations of the do-nothing baseline are 0.48 TER points (from 24.24 with "TER (pe)" to 23.76 with
"TER (pe+ref)") and 3.22 BLEU points (from 62.99 to 66.21). Interestingly, except for one system, all the results show larger variations when computed with "BLEU (pe+ref)", with a difference of 0.97 TER points (from 18.0 to 17.3) and 4.18 BLEU points (from 72.52 to 76.7 ) for the best system. Such variations are about 0.5 TER and 1.0 BLEU points larger than those measured for the baseline. This difference suggests that, though penalized by the comparison with human post-edits, a good amount of corrections made by the system still represent acceptable modifications of the original translations. Further analysis, which we leave for future work, should focus on understanding whether these corrections represent a problem (i.e. an unwanted deviation from the desired target style) or acceptable paraphrases of the input.

NMT subtask. Also in this case, as shown in Table 5, the multi-reference results computed with "TER/BLEU (pe+ref)" are better than those computed with "TER/BLEU (pe)". Apart from this, however, the performance variations for the baseline and the systems are not systematic nor particularly informative.

\section{System/performance analysis}

As a complement to global TER/BLEU scores, also this year we performed a more fine-grained analysis of the changes made by each system to the test instances.

\subsection{Macro indicators: modified, improved and deteriorated sentences}

Tables 6 and 7 show the number of modified, improved and deteriorated sentences, respectively for the PBSMT and the NMT subtasks. It's worth noting that, as in the previous rounds and in both the settings, the number of sentences modified by each system is higher than the sum of the improved and the deteriorated ones. This difference is represented by modified sentences for which the corrections do not yield TER variations. This grey area, for which quality improvement/degradation can not be automatically assessed, contributes to motivate the human evaluation discussed in Section 6.

PBSMT subtask. As can be seen in Table 6, the runs submitted to the PBSMT subtask reveal a quite homogeneous behaviour in terms of systems' aggressiveness. On average, the 11 submitted sys- 


\begin{tabular}{l|c|c|c} 
Systems & Modified & Improved & Deteriorated \\
\hline MS_UEdin Primary & $1,641(82.05 \%)$ & $1,111(67.70 \%)$ & $331(20.17 \%)$ \\
FBK Contrastive (MRT+MLE) & $1,581(79.05 \%)$ & $1,039(65.72 \%)$ & $319(20.18 \%)$ \\
FBK Primary (MRT) & $1,573(78.65 \%)$ & $1,025(65.16 \%)$ & $323(20.53 \%)$ \\
POSTECH Contrastive (fix5) & $1,577(78.85 \%)$ & $1,001(63.47 \%)$ & $342(21.69 \%)$ \\
POSTECH Primary & $1,566(78.30 \%)$ & $992(63.35 \%)$ & $338(21.58 \%)$ \\
POSTECH Contrastive (var5) & $1,565(78.25 \%)$ & $987(63.07 \%)$ & $341(21.79 \%)$ \\
USAAR_DFKI Primary & $1,435(71.75 \%)$ & $751(52.33 \%)$ & $469(32.68 \%)$ \\
USAAR_DFKI* & $1,595(79.75 \%)$ & $812(50.91 \%)$ & $548(34.36 \%)$ \\
DFKI-MLT Primary (Transf.large) & $1,221(61.05 \%)$ & $469(38.41 \%)$ & $457(37.43 \%)$ \\
DFKI-MLT Contrastive (Transf.base) & $1,157(57.85 \%)$ & $414(35.78 \%)$ & $445(38.46 \%)$ \\
DFKI-MLT Contrastive (LSTM) & $1,573(78.65 \%)$ & $567(36.05 \%)$ & $659(41.89 \%)$ \\
\hline
\end{tabular}

Table 6: Number of test sentences modified, improved and deteriorated by each run submitted to the PBSMT subtask.

\begin{tabular}{l|c|c|c} 
Systems & Modified & Improved & Deteriorated \\
\hline FBK Primary (MRT) & $276(26.98 \%)$ & $131(47.46 \%)$ & $77(27.90 \%)$ \\
MS_UEdin Primary & $316(30.89 \%)$ & $150(47.47 \%)$ & $107(33.86 \%)$ \\
FBK Contrastive (MRT+MLE) & $298(29.13 \%)$ & $134(44.97 \%)$ & $88(29.53 \%)$ \\
POSTECH Contrastive (top1) & $230(22.48 \%)$ & $105(45.65 \%)$ & $87(37.83 \%)$ \\
POSTECH Primary (fix5) & $224(21.90 \%)$ & $103(45.98 \%)$ & $85(37.95 \%)$ \\
POSTECH Contrastive (var5) & $220(21.51 \%)$ & $101(45.91 \%)$ & $85(38.64 \%)$ \\
USAAR_DFKI Primary & $304(29.72 \%)$ & $99(32.57 \%)$ & $138(45.39 \%)$ \\
DFKI-MLT Contrastive (Transf.base) & $468(45.75 \%)$ & $60(12.82 \%)$ & $351(75.00 \%)$ \\
DFKI-MLT Primary (Transf.large) & $448(43.79 \%)$ & $50(11.16 \%)$ & $342(76.34 \%)$ \\
DFKI-MLT Contrastive (LSTM) & $565(55.23 \%)$ & $51(9.03 \%)$ & $430(76.11 \%)$ \\
\hline
\end{tabular}

Table 7: Number of test sentences modified, improved and deteriorated by each run submitted to the NMT subtask.

tems modified about $75.0 \%$ of the sentences, with values ranging from $57.85 \%$ to $82.05 \%$. In line with last year's round, the top-performing ones are more aggressive (the best systems peaks at $82.05 \%$ modified sentences) than those in lowerranked positions. Since about $15.0 \%$ (i.e. 300) of the test instances are to be considered as "perfect" (see Figure1), the percentage of modifications is not too far to the expected value $(85 \%)$. However, in terms of precision (i.e. the proportion of improved sentences out of the total amount of modified test items), the average is only $54.7 \%$. While the three top submissions are able to improve more than $65.0 \%$ of the test items (with the best system peaking at $67.7 \%$ ), the lower-ranked ones do not exceed $53.0 \%$. The deteriorated sentences are on average $28.2 \%$, with only three systems that are able to limit this proportion to about $20.0 \%$. These results indicate that, although systems are able to change the expected number of sentences in the test set (with overall MT quality improvements, as shown in Table 4), their precision is still crucial. From this point of view, the room for improvement (more than 30 points in precision for the top submissions) remains large and advocates for solutions to drive APE technology towards the appropriate corrections (Chatterjee et al., 2018).

NMT subtask. In this subtask, the participating systems show a less aggressive behaviour and a tendency to preserve the higher quality of NMT translations. On average, the 10 submitted runs modified $32.7 \%$ of the sentences, with values ranging from $21.51 \%$ to $55.23 \%$. However, though desirable, this behaviour is too conservative. Considering that about $25.2 \%$ (i.e. 257) of the test instances are to be considered as "perfect" (see Figure2), the reported numbers are far below the target percentage of modifications $(74.8 \%)$. Also in terms of precision, the values are lower than in the PBSMT subtask. The average is $34.3 \%$ and even the top submissions have a percentage of improved sentences of less than $50.0 \%$. The same holds for the percentage of deteriorated sentences (the average is $47.85 \%$ ), for which all systems have larger values when dealing with neural outputs. Overall, the analysis confirms that correcting high-quality translations still remains a hard task, especially when dealing with NMT outputs. On 


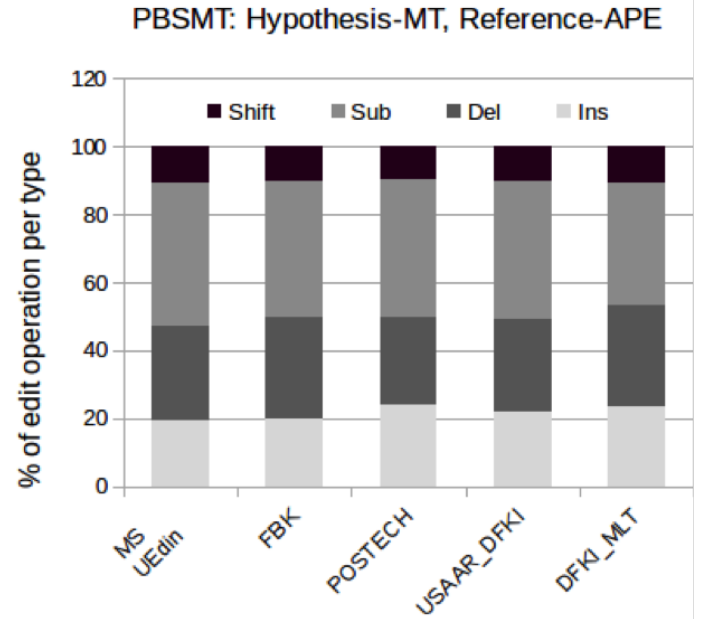

Figure 3: System behaviour (primary submissions) for the PBSMT subtask - TER(MT, APE)

one side, as we observed in the PBSMT subtask, systems' low precision is an evident limitation. On the other side, and in addition to that, neural translations might be particularly difficult to improve, even for neural APE models. Since NMT is known to produce considerably less reordering errors than PBSMT (Bentivogli et al., 2016), one possible explanation is that the margins of improvement to the input sentences are reduced to types of errors (e.g. lexical) on which APE systems are less reliable. The analysis proposed in Section 5.2 aims to explore also this aspect.

\subsection{Micro indicators: edit operations}

We now turn to analyze the possible differences in the way systems corrected the test set instances. To this aim, we looked at the distribution of the edit operations done by each system (insertions, deletions, substitutions and shifts) by computing the TER between the original MT output and the output of each system taken as reference (only for the primary submissions). The outcomes of this analysis are shown in Figures 3 (PBSMT subtask) and 4 (NMT subtask).

PBSMT subtask. As it is evident from Figure 3, little can be said about the small differences in system's behaviour. Indeed, the plot does not show noticeable differences between neural-based submissions that, in most of the cases, implement similar solutions (multi-source, Transformer-based models trained with the same in-domain and artificial corpora). All of them are characterized by a rather homogeneous distribution of the types of correction patterns applied, with a large number

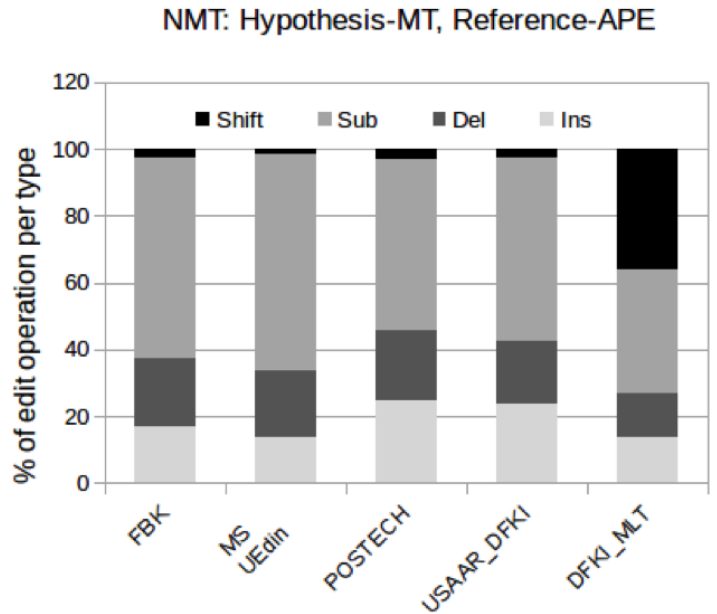

Figure 4: System behaviour (primary submissions) for the NMT subtask - TER(MT, APE)

of substitutions (average $39.8 \%$ of the total) and a slight dominance of deletions (average 28.2\%) over the others (average insertions and shifts are respectively $21.6 \%$ and $10.4 \%$ of the total).

NMT subtask. Also in this case, most of the submissions are characterized by a similar behaviour, probably induced by the slightly different solutions adopted by participants. The distribution of edit operations, however, is less homogeneous than in the PBSMT subtask. Substitutions still represent the majority of the corrections but with a larger percentage (average 53.5\%), which is followed by insertions (18.7\%), deletions $(18.5 \%)$ and shifts $(9.2 \%)$. Average values, however, are influenced by one submission (DFKIMLT), which shows a skewed distribution towards shift operations $(36.15 \%)$ that are close in percentage to substitutions $(36.88 \%)$. In terms of raw percentages, the role of shift operations can explain the lower performance of this outlier system, which was probably penalized by a large number of unnecessary reordering actions. As a more general observation, comparing Figures 3 and 4, we observe that reordering plays a quite different role in the two subtasks. Systems trained and evaluated on PBSMT data learn and apply more substitutions than those built for the NMT scenario. This can be explained by the higher fluency of neural translations which, among the four types of corrections, reduces the necessity of reordering operations. If this hypothesis holds true, the improvements of NMT outputs will mostly depend on other aspects like lexical choice, as suggested by the larger amount of substitutions compared to 


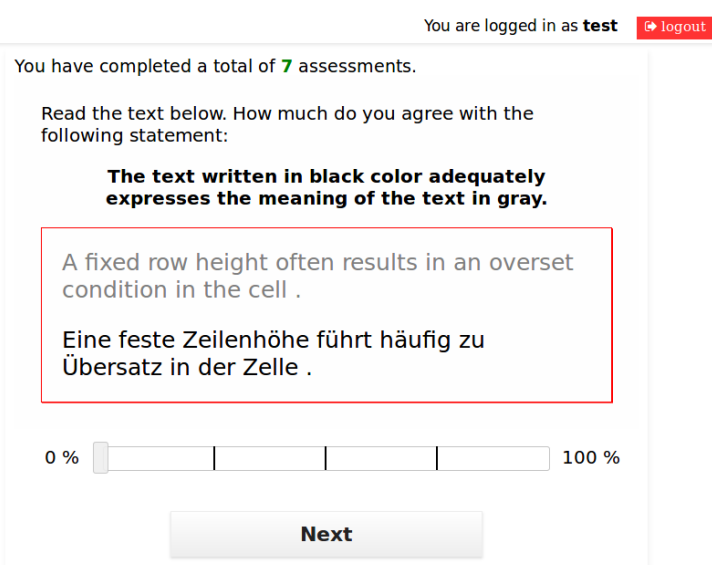

Figure 5: Screenshot of the direct assessment user interface.

the PBSMT subtask.

\section{Human evaluation}

In order to complement the automatic evaluation of APE submissions, a manual evaluation of the primary systems submitted (five in total) was conducted. Similarly to the manual evaluation conducted for last year APE shared task, it was carried out following the direct assessment (DA) approach (Graham et al., 2013; Graham et al., 2016). In this Section, we present the evaluation procedure as well as the results obtained.

\subsection{Evaluation procedure}

The manual evaluation carried out this year involved 12 native German speakers with full professional proficiency in English in the IT domain, with a third of the evaluators being students in translation technologies from Saarland University and the remaining ones researchers and engineers from DFKI. Each evaluator was introduced to the evaluation task through a set of slides and a testing phase of the evaluation platform in order to be familiar with the user interface and its functionalities. A screenshot of the evaluation interface is presented in Figure 5.

A single assessment consists in assigning a score to a German sentence indicating how much of the meaning from a source sentence in English is expressed. In other words, the adequacy of a translation is directly evaluated on a scale from 0 to 100 given the source. The evaluators are free to conduct as many assessments as they want and free to schedule their own evaluation sessions. In addition, there was no requirement regarding a minimum amount of assessments to perform. The evaluation took place over a period of a month and

\begin{tabular}{l|r|r} 
Subtask & PBSMT & NMT \\
\hline \# Systems & 7 & 7 \\
\# Source segs & 2,000 & 1,023 \\
\# Total Pairs & 14,000 & 7,161 \\
\# Unique Pairs & 8,749 & 2,916 \\
Reduction & $37.5 \%$ & $59.3 \%$ \\
\hline
\end{tabular}

Table 8: Data statistics per subtask with the total number of assessments prior to and after combination of identical target segments for each source.

was conducted in two sessions: a first one focusing on the PBSMT subtask and a second one on the NMT subtask.

For each subtask, the submitted post-edited test sets from the participants were presented to the evaluators one sentence at a time along with the corresponding source sentence. In order to define a baseline and an upperbound for this manual evaluation, the baseline (no post-edits) and the human post-edited MT output were added to the pool of submissions to evaluate, leading to a total of 14,000 and 7,161 pairs of segments to evaluate for the PBSMT and NMT subtasks respectively. However, it was possible to take advantage of the fact that multiple systems can produce identical outputs, allowing us to combine them and reduce the total number of source-target pairs to evaluate. Table 8 contains the statistics relative to the numbers of translations in total for all systems, as well as savings in terms of assessment effort that was gained by combining identical system outputs prior to running the evaluation.

Based on the direct assessment scores provided by the evaluators, two scores were computed for each system. A first score is the average of the segments direct assessment scores (noted "Avg $\%$ "). For the second score (noted "Avg $z$ "), human assessments for translations were first standardized according to each individual human assessor's overall mean and standard deviation score. Average standardized scores for individual segments belonging to a given system are then computed, before the final overall DA score for that system is computed as the average of its segment scores.

\subsection{Human Evaluation results}

The twelve human evaluators spent a total of 64 hours on the DA task with an average of 17.2 and 17.5 seconds per assessment for the NMT and PBSMT subtasks respectively. More details about the 


\begin{tabular}{r|rr|rr} 
& \multicolumn{3}{|c|}{ \# Assessments } & \multicolumn{2}{|c}{ Avg. Duration (sec.) } \\
ID & PBSMT & NMT & PBSMT & NMT \\
\hline 1 & 672 & 660 & 19.87 & 16.52 \\
2 & 420 & 93 & 19.56 & 24.79 \\
3 & 2,000 & 0 & 19.69 & - \\
4 & 1,153 & 228 & 20.57 & 23.38 \\
5 & 751 & 20 & 23.62 & 27.71 \\
6 & 1,500 & 200 & 16.58 & 15.60 \\
7 & 60 & 0 & 24.66 & - \\
8 & 2,401 & 300 & 10.90 & 5.59 \\
9 & 276 & 660 & 23.92 & 19.43 \\
10 & 0 & 668 & - & 20.67 \\
11 & 0 & 1,020 & - & 13.73 \\
12 & 0 & 100 & - & 30.27 \\
\hline
\end{tabular}

Table 9: Direct assessments statistics indicating the number of assessments carried out per subtask and the average duration in seconds per assessment for the twelve evaluators involved in the manual evaluation.

\begin{tabular}{|c|c|c|c|}
\hline \# & Systems & Avg \% & Ave $z$ \\
\hline & Human post-edit & 95.87 & 0.50 \\
\hline 1 & MS_UEdin & $\overline{93.2 \overline{7}}$ & $\overline{0} . \overline{4} 1^{-}$ \\
\hline$\overline{2}$ & $\overline{\mathrm{F}} \overline{\mathrm{B}} \overline{\mathrm{K}}$ & 90.80 & $0 . \overline{3} 3$ \\
\hline & POSTECH & 89.96 & 0.29 \\
\hline 4 & $\overline{\mathrm{US}} \overline{\mathrm{A}} \overline{\mathrm{A}} \overline{\mathrm{R}} \overline{\mathrm{D}} \overline{\mathrm{F}} \overline{\mathrm{K}} \mathrm{I}$ & $\overline{86.1 \overline{4}}$ & $0 . \overline{15}$ \\
\hline$\overline{5}$ & $\overline{\mathrm{D}} \overline{\mathrm{F}} \overline{\mathrm{K}} \overline{\mathrm{I}}-\overline{\mathrm{M}} \overline{\mathrm{L}} \overline{\mathrm{T}}$ & $\overline{77.7 \overline{8}}$ & $-\overline{0} . \overline{1} \overline{5}$ \\
\hline & Baseline & $\overline{75.9 \overline{2}}$ & $-\overline{0} . \overline{2} \overline{2}$ \\
\hline
\end{tabular}

Table 10: DA Human evaluation results for the PBSMT subtask in terms of average raw DA (Ave \%) and average standardized scores (Ave $z$ ). Dashed lines between systems indicate clusters according to Wilcoxon signed-rank test at $\mathrm{p}$ level $p \leq 0.05$.

assessments done per evaluator, as well as the average duration per assessment, are presented in Table 9 .

PBSMT Subtask. The results of DA for the PBSMT subtask are presented in Table 10. Six clusters are defined, grouping systems together according to which systems significantly outperform all others in lower ranking clusters based on the Wilcoxon signed-rank test. The human postedited MT output reaches an averaged DA score of $95.87 \%$, followed by the first system (MS_UEdin), single in a cluster and significantly better than the other systems, with an averaged DA score of $93.27 \%$. A second cluster contains two systems which are non significantly different reaching $90.9 \%$ and $89.96 \%$ averaged DA scores.

All submitted systems are ranked significantly higher than the baseline (MT output without postediting) but the top system remains below the hu-

\begin{tabular}{|c|c|c|c|}
\hline \# & Systems & Ave $\%$ & Ave 2 \\
\hline \multirow{7}{*}{1} & Human post-edit & 96.13 & 0.43 \\
\hline & $\overline{\mathrm{MS}} \overline{\mathrm{U}} \overline{\mathrm{E}} \mathrm{din}$ & 91.11 & $0 . \overline{2} 4$ \\
\hline & POSTECH & 90.41 & 0.22 \\
\hline & FBK & 90.41 & 0.20 \\
\hline & Basel & 90.18 & 0.20 \\
\hline & USAAR_DFI & 89.97 & 0.19 \\
\hline & DFKI-MLT & 89.53 & 0.18 \\
\hline
\end{tabular}

Table 11: DA Human evaluation results for the NMT subtask in terms of average raw DA (Ave \%) and average standardized scores (Ave $z$ ). Dashed lines between systems indicate clusters according to Wilcoxon signed-rank test at $\mathrm{p}$ level $p \leq 0.05$.

man post-edits with a difference of $2.6 \%$. The ranking of primary submissions for the PBSMT subtask is similar to the one obtained with the automatic metrics evaluation, where all primary systems were ranked above the baseline. For the DFKI-MLT system, TER indicates a nonsignificant difference with the baseline while DA scores leads to this system being significantly higher than the baseline.

NMT Subtask. The results of DA for the NMT subtask are presented in Table 11. Similarly to the results obtained with automatic metrics, the baseline is ranked above two and below three primary submissions. However, none of the submissions are ranked significantly higher or lower than the baseline according to DA scores and all five submissions are placed in the same cluster. The human post-edited MT output reaches an averaged DA score of $96.13 \%$, ranked above the first system (MS_UEdin) with an averaged DA score of $91.11 \%$.

The range of averaged DA scores for the NMT subtask is smaller $([89.53 ; 96.13])$ compared to the PBSMT subtask ([75.92;95.87]), which is observed in the results obtained with automatic metrics as well. This indicates a higher translation adequacy for the NMT subtask and is supported by the averaged DA score obtained by the baseline system (no post-edits). In addition, the human post-edited MT output reaches a higher averaged DA score for the NMT compared to the PBSMT subtask (similarly to automatic metrics results), which could indicate a higher overall translation quality of the final translation after manually post-editing the baseline NMT output compared to a baseline PBSMT output. However, more experiments involving larger test sets and a larger pool of evaluators are necessary to validate this obser- 
vation.

\section{Conclusion}

We presented the results from the fourth shared task on Automatic Post-Editing. This year, we proposed two subtasks in which the MT output to be corrected was respectively generated by a phrase system (PBSMT subtask) and by a neural system (NMT subtask). Both the subtasks dealt with English-German data drawn from the information technology domain. This evaluation round attracted submissions from five groups, who submitted eleven runs for the PBSMT subtask and ten runs for the NMT one. Participants' systems have a lot in common: they are all neural models based on the Transformer architecture, some of them are based on multi-source methods and they all took advantage of the synthetic corpora released as additional training material. Evaluation results reflect such similarities and the effectiveness of the proposed solutions: top submissions have very close performance which, on both subtasks, shows significant improvements over the baseline.

In short, the main findings of this year's round are the following:

- Besides the amount of training data (the training corpora for the two subtasks have different size), the task difficulty is proportional to the quality of the initial translations. In line with previous years, learning from (and testing on) lower quality data leaves more room for improvement.

- The output of PBSMT systems is easier to improve (gains are up to -6.24 TER and +9.53 BLEU points). Such gains reflect a tendency to model the post-editors' style learned from training data.

- The output of NMT systems is harder to improve by current neural APE technology (gains are up to -0.38 TER and +0.8 BLEU points). A general explanations is that NMT translations are of higher quality. More specifically, looking the corrections done by the systems, the small number of reordering issues calls for effective methods to handle other types of errors (e.g. lexical choice) on which current APE technology can still be improved.
- Synthetic data help in improving performance. In the PBSMT subtask, similar to the APE17 English-German task from a task difficulty standpoint, the synthetic data provided as additional training material contributed to further improvements over the baseline.

\section{Acknowledgments}

The manual evaluation conducted during the automatic post-editing task has been partially supported by the German Federal Ministry of Education and Research (BMBF) under the funding code 01IW17001 (Deeplee) and by the German Research Foundation (DFG) as part of SFB 1102 "Information Density and Linguistic Encoding". We would like to thank the evaluators who participated in the manual evaluation and Christian Federmann for helping in data preparation and results extraction (Federmann, 2012).

\section{References}

Dzmitry Bahdanau, Kyunghyun Cho, and Yoshua Bengio. 2014. Neural machine translation by jointly learning to align and translate. arXiv preprint arXiv:1409.0473.

Luisa Bentivogli, Arianna Bisazza, Mauro Cettolo, and Marcello Federico. 2016. Neural versus PhraseBased Machine Translation Quality: a Case Study. In Proceedings of the 2016 Conference on Empirical Methods in Natural Language Processing, pages 257-267, Austin, Texas, November. Association for Computational Linguistics.

Ondřej Bojar, Rajen Chatterjee, Christian Federmann, Barry Haddow, Matthias Huck, Chris Hokamp, Philipp Koehn, Varvara Logacheva, Christof Monz, Matteo Negri, Matt Post, Carolina Scarton, Lucia Specia, and Marco Turchi. 2015. Findings of the 2015 Workshop on Statistical Machine Translation. In Proceedings of the Tenth Workshop on Statistical Machine Translation, pages 1-46, Lisbon, Portugal, September. Association for Computational Linguistics.

Ondřej Bojar, Rajen Chatterjee, Christian Federmann, Yvette Graham, Barry Haddow, Matthias Huck, Antonio Jimeno Yepes, Philipp Koehn, Varvara Logacheva, Christof Monz, Matteo Negri, Aurelie Neveol, Mariana Neves, Martin Popel, Matt Post, Raphael Rubino, Carolina Scarton, Lucia Specia, Marco Turchi, Karin Verspoor, and Marcos Zampieri. 2016. Findings of the 2016 Conference on Machine Translation. In Proceedings of the First Conference on Machine Translation, pages 131-198, Berlin, Germany, August. Association for Computational Linguistics. 
Ondřej Bojar, Rajen Chatterjee, Christian Federmann, Yvette Graham, Barry Haddow, Shujian Huang, Matthias Huck, Philipp Koehn, Qun Liu, Varvara Logacheva, Christof Monz, Matteo Negri, Matt Post, Raphael Rubino, Lucia Specia, and Marco Turchi. 2017. Findings of the 2017 Conference on Machine Translation (WMT17). In Proceedings of the Second Conference on Machine Translation, Volume 2: Shared Task Papers, pages 169-214, Copenhagen, Denmark, September. Association for Computational Linguistics.

Rajen Chatterjee, Marion Weller, Matteo Negri, and Marco Turchi. 2015. Exploring the Planet of the APEs: a Comparative Study of State-of-the-art Methods for MT Automatic Post-Editing. In Proceedings of the 53rd Annual Meeting of the Association for Computational Linguistics), Beijing, China.

Rajen Chatterjee, M. Amin Farajian, Matteo Negri, Marco Turchi, Ankit Srivastava, and Santanu Pal. 2017. Multi-source Neural Automatic Post-Editing: FBK's Participation in the WMT 2017 APE Shared Task. In Proceedings of the Second Conference on Machine Translation, pages 630-638. Association for Computational Linguistics.

Rajen Chatterjee, Matteo Negri, Marco Turchi, Frédéric Blain, and Lucia Specia. 2018. Combining Quality Estimation and Automatic Post-editing to Enhance Machine Translation Output. In Conference of the American Association for Machine Translation (Research Track), New Orleans, LA.

Christian Federmann. 2012. Appraise: an open-source toolkit for manual evaluation of MT output. The Prague Bulletin of Mathematical Linguistics, 98:2536.

Yvette Graham, Timothy Baldwin, Alistair Moffat, and Justin Zobel. 2013. Continuous measurement scales in human evaluation of machine translation. In Proceedings of the 7th Linguistic Annotation Workshop and Interoperability with Discourse, pages 33-41, Sofia, Bulgaria, August. Association for Computational Linguistics.

Yvette Graham, Timothy Baldwin, Alistair Moffat, and Justin Zobel. 2016. Can machine translation systems be evaluated by the crowd alone. Natural Language Engineering, FirstView:1-28, 1.

Teresa Herrmann, Jan Niehues, and Alex Waibel. 2013. Combining Word Reordering Methods on different Linguistic Abstraction Levels for Statistical Machine Translation. In Proceedings of the Seventh Workshop on Syntax, Semantics and Structure in Statistical Translation, Altanta, Georgia, USA.

Chris Hokamp. 2017. Ensembling Factored Neural Machine Translation Models for Automatic PostEditing and Quality Estimation. In Proceedings of the Second Conference on Machine Translation, Copenhagen, Denmark, September.
Matthias Huck, Fabienne Braune, and Alexander Fraser. 2017. Lmu munich's neural machine translation systems for news articles and health information texts. In Proceedings of the Second Conference on Machine Translation, Volume 2: Shared Task Papers, pages 315-322, Copenhagen, Denmark, September. Association for Computational Linguistics.

Marcin Junczys-Dowmunt and Roman Grundkiewicz. 2016. Log-linear combinations of monolingual and bilingual neural machine translation models for automatic post-editing. In Proceedings of the First Conference on Machine Translation, pages 751758, Berlin, Germany, August.

Marcin Junczys-Dowmunt and Roman Grundkiewicz. 2018. Microsoft and University of Edinburgh at WMT2018: Dual-Source Transformer for Automatic Post-Editing. In Proceedings of the Third Conference on Machine Translation, Brussels, Belgium, October.

Marcin Junczys-Dowmunt, Roman Grundkiewicz, Tomasz Dwojak, Hieu Hoang, Kenneth Heafield, Tom Neckermann, Frank Seide, Ulrich Germann, Alham Fikri Aji, Nikolay Bogoychev, André F. T. Martins, and Alexandra Birch. 2018. Marian: Fast neural machine translation in $\mathrm{C}++$. In Proceedings of ACL 2018, System Demonstrations, pages 116-121, Melbourne, Australia, July. Association for Computational Linguistics.

Guillaume Klein, Yoon Kim, Yuntian Deng, Jean Senellart, and Alexander Rush. 2017. Opennmt: Open-source toolkit for neural machine translation. In Proceedings of ACL 2017, System Demonstrations, pages 67-72. Association for Computational Linguistics.

Philipp Koehn. 2004. Statistical Significance Tests for Machine Translation Evaluation. In Proceedings of EMNLP 2004, pages 388-395, Barcelona, Spain.

Matteo Negri, Marco Turchi, Rajen Chatterjee, and Nicola Bertoldi. 2018. eSCAPE: a Large-scale Synthetic Corpus for Automatic Post-Editing. In Proceedings of the Eleventh International Conference on Language Resources and Evaluation (LREC 2018), Miyazaki, Japan, May 7-12, 2018.

Santanu Pal, Nico Herbig, Antonio Krüger, and van Genabith Josef. 2018. A Transformer-Based MultiSource Automatic Post-Editing System. In Proceedings of the Third Conference on Machine Translation, Brussels, Belgium, October.

Kishore Papineni, Salim Roukos, Todd Ward, and WeiJing Zhu. 2002. BLEU: A Method for Automatic Evaluation of Machine Translation. In Proceedings of the 40th Annual Meeting on Association for Computational Linguistics, ACL '02, pages 311-318.

Daria Pylypenko and Raphael Rubino. 2018. DFKIMLT System Description for the WMT18 Automatic Post-editing Task. In Proceedings of the Third 
Conference on Machine Translation, Brussels, Belgium, October.

Rico Sennrich, Barry Haddow, and Alexandra Birch. 2016. Neural machine translation of rare words with subword units. In Proceedings of the 54th Annual Meeting of the Association for Computational Linguistics (Volume 1: Long Papers), pages 17151725, Berlin, Germany, August.

Rico Sennrich, Orhan Firat, Kyunghyun Cho, Alexandra Birch, Barry Haddow, Julian Hitschler, Marcin Junczys-Dowmunt, Samuel Läubli, Antonio Valerio Miceli Barone, Jozef Mokry, and Maria Nadejde. 2017. Nematus: a Toolkit for Neural Machine Translation. In Proceedings of the Software Demonstrations of the 15th Conference of the European Chapter of the Association for Computational Linguistics, pages 65-68, Valencia, Spain, April.

Shiqi Shen, Yong Cheng, Zhongjun He, Wei He, Hua Wu, Maosong Sun, and Yang Liu. 2016. Minimum risk training for neural machine translation. In Proceedings of the 54th Annual Meeting of the Association for Computational Linguistics (Volume 1: Long Papers), pages 1683-1692. Association for Computational Linguistics.

Jaehun Shin and Jong-Hyeok Lee. 2018. Multiencoder Transformer Network for Automatic PostEditing. In Proceedings of the Third Conference on Machine Translation, Brussels, Belgium, October.

Michel Simard, Cyril Goutte, and Pierre Isabelle. 2007. Statistical Phrase-Based Post-Editing. In Proceedings of the Annual Conference of the North American Chapter of the Association for Computational Linguistics (NAACL HLT), pages 508-515, Rochester, New York.

Matthew Snover, Bonnie Dorr, Richard Schwartz, Linnea Micciulla, and John Makhoul. 2006. A Study of Translation Edit Rate with Targeted Human Annotation. In Proceedings of Association for Machine Translation in the Americas, pages 223-231, Cambridge, Massachusetts, USA.

Lucia Specia, Kim Harris, Aljoscha Burchardt, Marco Turchi, Matteo Negri, and Inguna Skadina. 2017. Translation Quality and Productivity: A Study on Rich Morphology Languages. In Proceedings of the 16th Machine Translation Summit, Nagoya, Japan, September.

Amirhossein Tebbifakhr, Ruchit Agrawal, Matteo Negri, and Marco Turchi. 2018. Multi-source Transformer with Combined Losses for Automatic PostEditing. In Proceedings of the Third Conference on Machine Translation, Brussels, Belgium, October.

Ashish Vaswani, Noam Shazeer, Niki Parmar, Jakob Uszkoreit, Llion Jones, Aidan N Gomez, Ł ukasz Kaiser, and Illia Polosukhin. 2017. Attention is all you need. In I. Guyon, U. V. Luxburg, S. Bengio,
H. Wallach, R. Fergus, S. Vishwanathan, and R. Garnett, editors, Advances in Neural Information Processing Systems 30, pages 5998-6008. Curran Associates, Inc. 